\title{
'TA'TIANA CRIVELLI
}

\section{PAMELA, O LA METAMORFOSI RICOMPENSATA*}

\section{In liaggio}

Poche stagioni risultano tanto adeguate per riflettere sul concetto di metamorfismo testuale quanto il XVIII secolo, che - non fosse altro che per l'emergere di generi letterari radicalmente nuovi (dal giornalismo fino al romanzo cosiddetto "borghese") - può collocarsi tra quelle epoche che Cesare Segre descrive come "indenni o estranee alle classificazioni", e nel corso delle quali, dunque, "lo sviluppo dei generi può esser visto come la maturazione di tradizioni, l'istituirsi (per imitazione di modelli prestigiosi) di connessioni tra certi contenuti e certe forme espositiven (SEGRE 234). ${ }^{2}$ E, parlando di generi letterari in quest'epoca proteiforme, non si può trovare soggetto più intrigante di Pamela, la vergine virtuosa e schiva che ha goduto di una popolarità senza pari in tutta Europa, ${ }^{2}$ la romanzesca creatura nata da mano maschile ad incarnare un ideale di femminilità.

Figura stessa della trasformazione. Pamela è trasmigrata per oltre mezzo secolo da un genere allaltro (si veda in proposito la tavola cronologica). trascorrendo - prima di cadere nell'oblio riservatole dall'evoluzione del gusto - dalle pagine dei romanzi a quelle dei libretti d'opera, per approdare sui palcoscenici teatrali di capitali e province, in versi e in prosa di ogni lingua o quasi del continente: a partire dal 1756 ed entro il primo ventennio dell'Ottocento la sola versione goldoniana della Pamela venne tradotta in inglese e tedesco, in francese (1759), portoghese, spagnolo, norvegese, greco (1806), russo (1812) e boemo. Di fronte alle sue innumercvoli identità non viene allora da chiederle in quale, tra queste, si sia scontita più a suo agio? Insomma: a chi appartiene Pamela?

Incontrando questa figua nell ambito di un più ampio studio sul romanzo del secondo Settecento italiano. ${ }^{3}$ mi è parso che questa losse l'occasione per dedicarle almeno una parte di quelle attenzioni che credo meriterebbe. Con particolare riguardo per la rielaborazione goldoniana della figura creata originariamente da Samuel Richarctson, (cfr. punti f. e m. della talvola) propongo qui una riflessione "di genere": e il termine si connetta pure - in tutta lá sua moderna ambiguità, peraltro giustilicata già clal suo etimo látino - sia 
allidea di stirpe d’appartenenza, sia a quella di gender.

\section{Dall Ingbilterra all'Italia}

Sin dal suo apparire, Pamela porta con sé dei tratti specifici che la connotano come rivoluzionaria: e perché l'aggettivo non sembri enfatico basti ricordare che è proprio con il diffondersi delle traduzioni e delle imitazioni dell'omonimo testo di Richardson che si inaugura l'"anglomania" continentale ed entra in Europa quel nuovo gusto per la narazione romanzesca che avrebbe messo in dicussione i canoni letterari tradizionali. Ma si potrebbe ad esempio aggiungere, sempre a giustificare laggettivo, che è con la Pamela che Goldoni mette in scena la prima commedia senza maschere della sua "riforma", o ricordare che proprio la rappresentazione di una versione francese della pièce goldoniana presso la Comédie Française (quella tradotta da Neufchâteau, punto o. della tavola), si concluse con l'arresto dell'intera troupe e dell'autore: stavolta per non essere invece (siamo nel 1793) sufficientemente "rivoluzionaria". Una sequela di eventi esterni, dunque, che ci lascia intuire come l'oggetto del discorso veicoli di per sé aspettative e contenuti fortemente incisivi, e ci rende curiosi di indagarne l'eventuale persistenza attraverso le diverse forme assunte dal soggetto.

Dei contenuti, è presto detto: il romanzo di Samuel Richardson, i cui clue primi toni compaiono anonimi a Londra nel 1740 (cfr: tavola, punto 1) narra in forma epistolare (la narratrice-protagonista scrive ai genitori, contadini, dalla casa nobiliare in cui è impiegata come cameriera) della storia dei suoi rapporti con il giovane nobiluomo presso cui si trova ad essere in servizio: alla morte di sua madre, protettrice di Panela, l'ereditiero Milord B. s'incapriccia della giovane e dà arvio a tutta una serie di tentativi di seduzione che, costantemente e fermamente respinti dalla virtuosa quindicenne, culminano in un rapimento. Trattenuta in una casa di campagna da cui cerca di fuggire rischiando la vita e salvandosi solo grazie ad un provvidenziale svenimento da un tentativo di stupro, Pamela oppone un netto rifiuto all'idea di diventare la mantenuta di milord. E a forza di buone ragioni e di implacabili rifiuti, ottiene lá libertà. Ma solo per tornare sui suoi passi e portare a conpimento lá redenzione del libertino: il romanzo si conclude con il matrimonio trá i due protagonisti e il pubblico riconoscimento clel merito di Pamela, elevata ad un rango sociale a cui, tradizionalmente, nessuna cameriera o figlia di contadini avrebbe potuto aspirare."

Pare chiaro, anche dá questa minima parafrasi riassuntiva, che il románzo infrange le consuetudini dell Europa del tempo: il matrimonio tra il nobile e la ragazza di origine contadine può clirsi un vero e proprio affronto alle bar- 
riere sociali vigenti, che eleva le qualità morali a unicá considerazione di merito. Lo stesso Richardson sembra voler moderare la portata provocatoria di tale matteria, che suscitò del resto varie reazioni all’apparire del romanzo. con il seguito della sua Pamela, (Pamela in ber Evalled Condition, clir. tárolat al punto la.), sottolineando l'assoluta eccezionalita del caso narato e delle qualità della protagonistá. La prima Pamela contiene infatti osservazioni piuttosto chiare sui rapporti tra nobilta e popolo. per capire la forza dei toni usati nella narrazione bastino la lettera scritta da Lady Davers al frátello Mr. B., nel momento in cui questa viene messa al corrente dei progetti matrimoniali, e il commento che a tali pensieri offre Pamela:

Quanto al matrimonio mi arrischio a credere che non ci pensiate veramente [...]. Mi è stato suggerito, ciononostante, da altri, che il vostro orgoglio contenga bassezza sufficiente per pensare a una cosa simile, tanto stregato siete. a quanto pare. da questa ragazza. Questa illazione. per quanto infondata. mi allarma molto. Riflettete. fratello, che la nostra non è una famigliá di parlenus. È antica quanto le migliori del regno, e da parecchie centinaia di anni non si è mai saputo che i suoi eredi si siano disonorati con matrimoni diseguali [...]. Se discendeste da una famiglia di ieri, dá una non più lontaná di una generazione o due da quel fango che tanto sembra attirarvi, la cosa sarebbe diversa. Lasciate che vi dica che io e tutti i miei vi disconosceremo per sempre se potrete abbassarri così. (RichaRdson 1995, 33t). ${ }^{5}$

\section{E Pamela reagisce così:}

Che letrera, miei cari padre e madre! Da essa si può redere come sia disprezzata la povera gente dai ricchi e dai potenti! E tuttavia eravamo tutti alla pari in origine: certo questa gente orgogliosa non pensa mai a che tappa breve sia la vita: né che. con tutta la loro vanità, verrà un tempo in cui saranno sulla stesso piano nostro. (Richardsox 1995. 335). ${ }^{6}$

Se l'intento dichiarato del primo autore era infatti altamente moraleggiante, proponendosi Richardson di "divertire e intrattenere, e allo stesso tempo istruire e migliorare gli animi dei giovani di ambo i sessi; [...] inculcare la religione e la moralità [...]: proporre nellá luce più esemplare i doreri dei genitori, dei figli, e quelli sociali. [...] dipingere il vizio nei suoi colori appropriati. sì da renderlo meritatamente odioso: e collocare la virtù nella sua luce amabile, per farla apparire leggiadra" (Prefazione del curatore. Richardsion 1995, ix) la questione della mésalliance finale e clel passaggio di classe di Pamela colpisce in manicra diretta la comune sensibilità ${ }^{8}$ e la strarolge in modo forte, anche se progressivo e convincente. nel trascorrere delle pagine del romanzo. 
Tuttavia "'intento morale dell'autore inglese [...] non conveniva né ai costumi né alle leggi del mio paese. A Londra un lord che sposi una contadina non deroga dagli impegni della nobità; a Venezia un patrizio che sposi una plebea priva i suoi figli del titolo nobiliare e toglie loro il modo di godere dei cliritti che le alte cariche possono conferiren: cosi Goldoni, nelle Memorie (Goliont 1994, II ix 278). Abbandonato un primo progetto di riduzione teatrale del romanzo di Richardson, perché esplicitamente in conflitto con i suoi icleali artistici ("La commedia, che è o dovrebbe essere la scuola dei costumi non deve esporre le debolezze umane se non per correggerle", GoLdoni 1994, II ix 278), Goldoni vi pose finalmente mano nel 1760. Ma in che modo restituire lo spirito e gli argomenti del romanzo inglese (che Goldoni ammette di afferrare "Senza imbarazzo") e non contraddire i propri principi artistici e la propria visione educativa dell'arte? Intervenendo sui contenuti, è orrio. ma anche sfruttando al meglio le diverse potenzialità del genere scelto per il proprio rifacimento: la commedia. Pure attenendoci soltanto a quanto scrive Goldoni netle Memorie dobbiamo rilevare almeno due fondamentali interventi sul testo:

\footnotetext{
Non mi áccinsi però al lavoro se non dopo aver escogitato uno scioglimento che, ben lungi dallessere pericoloso, potesse servir di modello agli amanti virtuosi, e rendere nello stesso tempo lá conclusione più piacevole e interessante. (Goldon1 1994, II ix 278).
}

Il trătto più saliente dell'intervento goldoniano a livello contenutistico. concerne dunque lintroduzione di unagnizione finale, che - come è noto srela le nobili origini di Pamela (il padre Andreuve, che aveva vissuto da contadino, cra in realtà "il conte d'Auspingh, scozzese, che durante le rivoluzioni della Scozia, perseguito come ribelle alla Corona britannica, aveva trovato rifugio sui monti d"Inghilterra". GoLdoni 1994. II ix 280). La seconda innovazione attiene invece più strettamente al genere: la sua prima commedial senza maschere è innovativa anche per altri versi essendo infatti, per ammissione dello stesso autore, piuttosto un dramma secondo la definizione dei francesi" (Goldoni 199+, 281):9 qualcosa dunque che costituisce, sempre seconclo le parole dello stesso Goldoni (GOLDONi 1994. II iii 256) "un genere di rappresentazione che sta tra la commedia e la tragedian. ${ }^{10}$

Ora, i pochi interventi critici che si occupano della Pamela italiana - pochi soprattutto perché a tuttoggi lá Pamela non è collocata clalla critica tra le commedie meglio riuscite di Golcloni, malgrado il fatto che al suo apparire "questa Commedia fu applauditissima, e furono ricercate le reppliche in ogni luogo dove si portò la Compannia. In Venezia, fra l'Autunno e il Carnovale 
del 1750, fu replicata dieciotto sere. e prima dellestate si fece per la primat volta in Milano", come si legge al tomo $V$ clelledizione Bettinellill - ne indagano assiduamente l'innovazione contenutistica. valutandola per la sua dose di reazionarismo rispetto all'impianto del romanzo inglese. e tratendo da ciò conclusioni in merito all ideologia del veneziano. 12 Il finale con Pamela nobile porrebbe, accanto ad un Goldoni pre-rivoluzionario, percepibile nel tono generale del discorso. un Goldoni conservatore vecchio stile; e in effetti. se Bonfil (il corrispettivo di Milord 13. nella commedia) può in quanto nobile esclamare: "Ah che la virtù di Pamelá clovea farmi arvertito che abbietto il di lei sangue non fosse!n (Pamela fancinlla, atto III. scena 11. Goldoni 1995, 171) questa affermazione convive nella commedia con passággi di tutt'altro tono:

Madama Jerre: "Che sabbia a morire per salvar l'onore. l'intendo; ma che sia disonore sposare una porera ragazza onesta. non la capisco. Io ho sentito dir tante volte che il mondo sarebbe più bello, se non l'avessero guastato gli uomini. i quali per cagione della superbia, hanno sconcertato il bellissimo ordine della natura. Questa madre comune ci considera tutti eguali. e l'alterigia dei grandi non si degna dei piccoli. Ma verrì un giomo, che dei piccoli e clei grandi si farà novamente tuta una pastan. (Pamela fanciulla. atto III, scena 3. Goldovi 1995, 155).

Si è dunque parlato dełle "due facce" di Goldoni, 13 e cercato cli sanare la contraddizione calando la produzione nel suo contesto storico-sociale (i matrimoni interclassisti, numerose testimonianze ce lo confemano, non erano di fatto ben accetti a V'enezia). ${ }^{1+}$ Ma nessuno ha posto attenzione specifica, in questo passaggio dal romanzo alla commedia, alla questione del genere: a questo livello Goldoni opera invece. a mio arviso, le trasformazioni più significative sulla vicenda, trasformazioni che hanno influsso diretto persino sullo svolgimento della trama.

\section{Dal romanzo al palcoscenico}

Innanzitutto, Goldoni trasporta la fruizione della vicenda da una sfera privata, come era per antonomasia quella della lettura nel secondo Settecento. ${ }^{15}$ ad una pubblicá, che segue duncpue modalità espressive di altro tipo. La dimensione teatrale implica forzatamente una considerazione più immediatá dellopinione del pubblico e un bellesempio di come Goldoni sfrutti questo tratto costitutivo del genere commedia è datto dalla creazione del personaggio di Nilord Artur: assente nel romanzo inglese, egli dí roce alla platea con argomentazioni sempre legate alla "ragione comune": se. ad 
esempio, le riflessioni sul comportamento libertino del protagonista maschile erano, nel romanzo, rese note attraverso la saggia mediazione della scrivente - Pamela - ecco che gli stessi pensieri, quando debbano essere trasportati sulla scena, si trasformano necessariamente in dialoghi o monologhi che, in quanto tali, non possono risultare eccessivamente carichi di considerazioni "teoriche" senza nuocere al ritmo dell'azione. Così Goldoni dà spazio alle ragioni clel buon senso non attraverso la minuziosa analisi dei fatti e clelle loro implicazioni (come si trova nel romanzo), ma portando sul palcoscenico la roce clell'opinione pubblica, nella concreta e stimabile persona di un nuovo personaggio: milord Artur, appunto. A conferma di quanto cletto si consideri l'impressionante serie di sentenze che Artur snocciola, a partire dalla sua prima comparsa in I, 13: "Gli anni passano. Non riserbate alla sposa l'età men bella. Chi tardi si marita. non vede si facilmente l'avanzamento de' suoi figliolin; alla domanda se un nobile debba necessariamente sposare una clama risponde: "tutte le buone regole insegnano che deve farsi" ma ammette con il consueto buonsenso che "non vi è regola che non patisca eccezione"; trova che la prudenza consista "nel vivere onestamente: nellosservare le leggi: nel mantenere il proprio decoro" e infine obietta all'unione di Bonfil e Pamela "sul fondamento della comune opinione" ossia del "modo di pensare degli uomini" (Pamela fanciulla, atto I, scena 13. Goldoni 1995, 99-104).

E a completare il quadro basti qui osservare. a titolo di esempio, come nella scena successiva (I, 14) le sue battute centrali - tutte di un solo perioclo ciascuna - siano, in sequenza, tre vere e proprie massime, di soggetto impersonale e rema generico: "Chi non esce clal suo paese rive pieno di pregiudizi", "Col viaggiare i superbi diventano clocili" e "Certamente, il mondo è un bel libro, ma poco serve a chi non sal leggere" (Pamela fancinlla, atto I, scena It. Goldoni 1995, 105).

\section{Dalla descrizione all azione}

Unaltrá fondamentale differenza di cui Goldoni cleve tener conto nel trachure in commedia il romanzo inglese, è quella dei tenmpi e dei luoghi: se, mantenendo lambientazione inglese clata cla Richardson allazione, ${ }^{16}$ egli parrebbe intervenire sui luoghi soltanto nella misura richiesta dall'uso settecentesco della traduzione - che tuttavia, è bene ricordarlo, richiede di norma un adattamento al carattere "nazionale" e implica e giustifica interventi anche macroscopici sul testo traslato - la situazione è ancora più complessá nel caso della ráaduzione della dimensione temporale del testo. Nel romanzo l'azione viene preparata con agio e si sviluppa nellarco di due lunghi tomi ( 650 palgine nella moderna edizione italiana, contro le 100 scarse 
della commedia goldoniana), cosi che Nitord B. può convincentemente procedere verso una lenta e sofferta conversione dal suo libertinaggio e Pancla può piano piano sentir nascere amore per lui; Goldoni, invece, deve ricondurre il tutto in tre atti di commedia, tenuti entro pochi interni: la cqual cosal lo farà sospirare de "E troppo malagevole impegno restrignere in poche ore una Favola, a cui si e clata dal primo Autore una estensione di mesi ed annin (L'Allore a cbi legge, in Pamela fancialla. Gomoni 1995, 78-79). Perció non è affatto un cáso che lá commedia goldoniana si apra si in maniera del tutto analoga al romanzo (Pamela che piange la morte dellá sua generosa padrona), ma lasci immediatamente capire come la giovinetta sia già innamorata di Bonfil: alla prima scena del primo atto Pámela in lacrime sospira augurandosi che "il cielo dia al mio padrone tuto quello che egli desidera" e assicurando, due battute oltre, che "egli ha il piü bel cuore del mondo". Bonfil, dal canto suo, le regala un anello sin dalla terza scena, per poi dichiarare già all'inizio della quarta, ex abrupto: "Jevre, io amo Pamela". Egli abbandona quasi immediatamente progetti di ordine extraconiugale e. soprattutto, non cerca di sopraffare la giovane con la violenza, limitandosi ad un tentativo di corruzione: sin clal primo atto, comunque, appare chiaro che ha già pensato al matrimonio. ${ }^{17}$ Lazione goldoniana perde dunque, a causa del passaggio in commedia, l'occasione di uno svolgimento lento e di un approfondimento dei rapporti tra i due personaggi: presentandoci Pamela e Bonfil reciprocamente innamorati, Goldoni sposta inevitabilmente il baricentro della vicenda che, da narrazione di una vittoriosa conversione di un libertino incallito per mezzo della virtù fatta donna, si trasforma piuttosto in un conflitto tra ragioni del cuore, che vorrebbero sposati i due innamorati. e ragioni sociali, che non reputerebbero la cosa affatto conveniente. Questa impostazione en raccourci fa si che al centro della commedia non ci siano più due storie indivichali che interagiscono, ma piuttosto le alterne vicende di una coppia di innamorati, che si presentano tali sin dall’inizio. E ciò implicar necessariamente una riduzione dello spessore psicologico delle due individualita: a soffrirne maggiormente, in rapporto al romanzo in cui era il perno centrale, è proprio la figura di Pamela.

\section{Da alltrice a personaggio}

Bisogna infatti ricordare, e qui entra in gioco un altra importante caratteristica relativa allat questione dei generi, che peculiare del romanzo richardsoniano cra la scelta della forma epistolare: e tale formula, mescolata a quella diaristica, non solo permetteva a Panela, attraverso minuziosissime descrizioni, di analizzare ed esporre (e in ultima analisi di gestire) gli even- 
ti in cui si imbatteva, ma la rendeva protagonista assoluta della trama. Proprio le sue lettere, infatti, fanno la Pamela narratrice del romanzo "autrice" del proprio destino e di quelli narrati: Milord B. ne è tanto consapevole che, per convincerla a mostragliele, afferma significativamente:

Poiché [con la mia persona, Ndr.] ti ho fornito un argomento, penso di avere il diritto di vedere come lo tratti. E poi, c’è un'aura romanzesca così graziosa nel cone tu racconti la tua storia, nei tuoi stratagemmi, e nei miei stratagemmi, che tanto meglio mi guiderà a organizzare la catastrofe clel bel racConto (RICHARDSON 1995, 297).18

Inoltre è proprio il modo in cui Pamela scrive che per primo affascina Milord B.: "Non ti ha certo nuociuto [...] che io abbia visto le lettere a cui alludi, poiché mi hanno dato uniopinione molto alta di te" (RICHARDson 1995 , 294). Non è possibile, per Goldoni orviare alla mancanza di questo mezzo stilistico narrativo sullá scena ${ }^{19}$ se non togliendo centralità alla figura di Pamela, tale quale è rappresentata nel romanzo epistolare; i brevi monologhi della Pamela italiana possono supplire solo in parte alla riduzione della prospettiva soggettivistica:

Pamela, sola: "Oh caro anello! Oh quanto mi saresti più caro, se dato non mi ti avesse il padrone! Ma se a me dato non l'avesse il padrone, non mi sárebbe sì caro. Egli acquista prezzo più clalla mano che me lo porse, che dal valor della gioia. Ma se chi me l'ha dato è padrone. ed io sono una povera serva, a che pro lo riceverò? Amo che me l'abbia dato il padrone, ma non vorrei ch'egli fosse padrone. Oh fosse egli un servo, come io sono, o foss'io una dama, com'egli è Cavaliere! Che mai mi converrebhe meglio desiderare? In lui la viltà o in me la grandezza? Se lui desidero vile, commetto una ingiustizia al suo merito; se bramo in me la grandezza, cado nel peccato dell'ambizione. Ma non lo bramerei per la vanità del grado. So io il perché, lo so io. Má sciocca che sono! Mi perclo a coltivare immagini più stravaganti dei sogni. Penso a cose che mi farebbero estremamente arrossire, se si sapessero i miei pensieri. Sento gente. Sarà madama Jevre». (Pamela fanciulla, atto I. scena 5. Goldow 1995, 88-89).

A causa del cambio di genere, cade dunque l'identificazione - che ha implicazioni fondamentali nel romanzo - tra lio narrante e la protagonista (identificazione che si proiettava a sua volta, ricreandone di nuove e più intriganti, sulla figura autoriale da un lato e quella dei destinatari dallaltro).

\section{Da sedolla a immamorata}

La tradizione teatrale italiana impone poi ulteriori aggiustamenti dell'im- 
magine della protagonista: abituato ad un archetipo femminile stilizzato e ben circoscritto dalla tradizione delle maschere, il pubblico italiano si attendeva sulla scena ruoli fissi e convenzionali: anche la Pannela golcloniana risente della modellizzazione prececkente, e viene abilmente ricondotta ad una figura gia familiare al pubblico: quella dell’innamorata. Di tale figura si può dire, come há fatto Clotilde Bertoni, che essà è, d'abitudine, "inserita in un intrigo di cui non maneggia i fili, deve rigorosamente attenersi ad un codice comportamentale che le preclude un’autonoma capacità d’azione. Né le è permesso di rivalersi sul piano linguisticon. ${ }^{20}$ All'innamorata della commedia clell'arte è infatti concessa una gamma di espressioni non molto vasta, né colorita (perlopiù discorsi stilizzati ed aulici) secondo la norma per cui i ruoli femminili, anche quelli di maggior peso, non sono posti al centro del discorso, se non quando si tratti o di cáratteri ridicoli o di tipi negativi. Nel teatro di Goldoni e. ancora di più, forse, in quello del suo acerrimo rivale l'abate Chiari, (autore, con la sua Pamela maritala del 1753, del primo seguito italiano dedicato alla commedia di Goldoni - cfr. tavola al punto g. - e a sua volta probabile ispiratore clella "maritata" goldoniana) la figura femminile guadagna invece uno spazio ben più importante, e proprio per diretto influsso del ruolo che le viene in quegli anni attribuito nei romanzi.21 Tuttavia non è in questo primo esperimento goldoniano che lá protagonista si libera dal suo ruolo stereotipato per splendere sulla scena: Pamela è ancora lontana dai profili autonomi di una Giacinta (nella famosa Trilogia della l'illeggiatura) o di una Mirandolina (La Locandiera). Del resto lo stesso Bonfil, imprigionato a sua volta nel ruolo di coppia, recupera in Goldoni molto spazio testuale a scapito cli Pamela ( 539 battute contro 313$)^{22}$ e finisce per ricalcare larchetipo del "primo amoroso": è la coppia di innamorati, nella linea della tradizione della commedia italiana, a costituire il soggetto della commedia.

Anche da un punto di vista stilistico, clunque, l'espressione della Pámela goldoniana non può che essere diversa da quella della sorella inglese: più matura e ponderata, meno passionale ed immediatamente diretta, più obbediente e dunque meno emozionale e veemente nell'espressione e nelle scelte linguistiche. ${ }^{23}$ Il tono diretto e deciso della Pamela di Richardson si oppone a quello mite e argomentativo della Pamela goldoniana: reagendo al primo tentativo di seduzione da parte di Mr. B. / Bonfil (operato nel romanzo attraverso un contatto fisico e, a lenire l'offesa, l'offerta di soldi, e nella commedia invece soltanto tramite l'offerta di clenaro) le clue protagoniste adottano un linguaggio dai toni decisamente diversi. In Richardson il dialogo è il seguente: 
"Come, sfrontatella! Ma lo sai con chi stai parlando?" Io ho perso ogni paura e ogni rispetto, e ho detto: "Si, lo so, signore, anche troppo bene! Ma posso dimenticare di esservi serva, se voi dimenticate come si deve comportare un padrone". "Che sciocca sfrontatella sei!" ha detto lui "Ti ho forse fatto del male?" "Sì, signore", ho detto io, "il più gran male del mondo: mi avete insegnato a climenticare il mio posto e la mia condizione; e avete accorciato la distanzal che la sorte ha messo fra di noi con l'abbassarvi fino a prendervi delle libertà con una povera serva. Eppure, signore, mi azzardo a dire che sono onesta, seppur povera: e, foste anche un principe, non vorrei essere altro che onestan. (Richardson 1995, 20).24

In Goldoni la scena si svolge allatto I, scena 6, e il prolisso intervento di Pamela inizia con queste parole, incomparabilmente meno salaci di quelle citate sopra:

Signore, io sono una povera serva, voi siete il mio padrone. Voi Cavaliere, io nata sono una misera donna: ma due cose eguali abbiam noi, e son queste la ragione e l'onore. Voi non mi darete ad intendere d'aver alcuna autorità sopra l'onor mio; poiché la ragione mi insegna esser questo un tesoro indipendente da clıi che sia. Il sangue nobile è un accidente della fortuna; le azioni nobili caratterizzano il grande. Che volete, signore, che dica il mondo di voi, se vi abbassate cotanto con una serva? (Pamela fanciulla, atto III, scena 6. Goldon 1995, 92).

Calata nel ruolo dell'innamorata, la stessa sua virtù viene intesa in modo diverso e, soprattutto, meno verboso: "Non intendo quella virtì eroica che commuove con le sue calamità e fa lagrimare con i suoi discorsi" scrive Goldoni a proposito di quella versione "in incognito" di Pamela che è la Putta onorata (Goltooni I994, parte II, cap. III, 256):25 la sua Pamela vuole "guadagnare i cuori con le attrattive clella virtù" piuttosto che "con l'orrore clel vizio" (GoldoNi 1994, parte II, cap. III, 256), e dunque avrà più bisogno di clare buoni esempi che non di predicare contro gli errori. Del resto, se è vero che Pamela piacaue al pubblico italiano, secondo la valutazione che ne dà lo stesso autore, proprio per essere una commedia contenente "più interesse e più azione" rispetto alla Pamela maritata che, al contrario, faceva mostra di "più studio e più arten (GoLdoni 1994, parte II, cap. XXXVIII, 407)26 sono proprio i tratti ora elencati della resa linguistica e morale del personaggio che vengono, con la ben nota arguzia, colti e "frustati" da Baretti. Senza mezzi termini egli dice la Pamela goldoniana "una sciocca cianciera, una pettegola volgare, una ciarlatana noiosa" ${ }^{-}$e, più oltre, "una pettegola e una pinzochera [...] lontana mille miglia dal partare e dall'operare come daman avendo in bocca "rocaboli e frasi sempre plebee" (BARETT! 179 e 183).28 


\section{Da sema a nobildomna}

Lo spostamento dell attenzione dalle clue figure in opposizione (femminile e maschile) alla coppia in sintonia, catusato dal nuovo genere d'appartenenza (la commedia), provoca necessariamente una caduta di tensione e l'analisi tipologica può dunque offrire una nuova chiave di lettura anche all primo fondamentale cambiamento goldoniano, valutato sinora esclusivamente da un punto di vista attenente al contenuto: l'agnizione finale. 29 Il nuovo soggetto, la coppia, non può infatti far altro che attendere una soluzione esterna al proprio problema, derivante appunto dalle regole sociali: ed ecco dunque che il clare a Pamela dei nobili natali risolve brillantemente il meccanismo teatrale e si rivela una soluzione che non ra letta solo sul piano del contenuto (reazionario o meno), ma si inserisce perfettamente nella logica imposta al testo dal suo essere commedia. Ed è con giusto orgoglio di conoscitore dei meccanismi interni al genere che Goldoni può scrivere. nella prefazione alla sua commedia, che "rade volte succede ciò che a me questa fiata è riuscito, di valersi dei caratteri solamente, e prendendo clella favola il buono. raggirar la catastrofe con un pensier nuovo, e rendere lo scioglimento più diletterole" (Pamela fanciulla. GoldoNi 1995. 79).

Insomma: trasportare Pamela dal romanzo alla commedia, dalle pagine del libro al palcoscenico, ne ha modificato inevitabilmente il destino, conferendole un esistenza parallela, tra le molte che il secolo le avrebbe riservato. E allora: a chi appartiene Pamela? Forse si potrebbe affermare, con la maschia espressione usata da Pietro Toldo, che Pamela è "un po" roba di tuttin (Toldo 344); eppure. vista da qui e in compagnia del reneziano. sembrerebbe proprio un altra donna...

\section{Unicersità di Zurigo}

\section{NOTE}

- Questo articolo rielabora, tenendo conto dei contributi apportati dal pubblico alla discussione, alcune riflessioni da me esposte in occasione del XVI Convegno Internazionale AISLLI, svoltosi presso la L'niversity of California di Los Angeles dal 6 al 9 ontobre 1997. i cui Atti sono tuttora in corso di stampa.

1 Particolarmente per periodi storici come quelli descritti sopra. allora. "la definizione dei generi sarà un modo per prendere atto, criticamente. della muterole storia di queste 
connessioni. che costituisce il quadro in cui si sviluppa l'attività letteraria" (SEgRE 234).

2 Sulla popolarità europea del tema (da Richardson al Voltaire di Nanine) i contributi critici sono decisamente più numerosi degli studi concernenti le "Pamele" italiane: per capire la molteplicita delle forme della ricezione europa del personaggio (letterarie e non) basterà consultare il fondamentale articolo di Grantham TLRNER. La migliore visione d'insieme, relativa alla diffusione e alla ricezione della pièce goldoniana, si trova nella Nota sulla fortmana del volume dell'edizione nazionale GoLDON 1995 (da cui sono tratte qui tutte le citazioni della Pamela). Per un panorama descrittivo delle edizioni circolanti nell Italia del Settecento restá ancora fondamentale la consultazione del volume di MARCHFsi, 18-20 e 404 in particolare. La dimensione teatrale italiana è invece rievocata con puntualità nell'articolo di Comparsin. Lá ricezione francese della Pamela goldoniana è stata studiata da JONARD 1965.

3 Lo studio, clal titolo prorvisorio di "Né Arturo. né Tumpino, né la Tavola Rotonda": romanzi del secondo Settecento italiano" è attualmente in corso di stampa presso la Salerno Ed., Roma, per la Collana "Studi e Saggi".

+ Sul tema di Cenerentola, intesa come archetipo femminile per la narrazione della promozione sociale, sono numerose le scritture critiche interessanti. Con particolare profitto per il nostro argomento si leggano FOLKENFuK e CECION1 75-78.

5 Il testo inglese recita: "As to marriage, I dare say you don't think of it. [...] It has been hinted to me, nevertheless, by others, that you have meanness enough in your pride, to think of such a thing: so bewitched are you, it seems, by this girl. This, though I think it must be a groundless sumise, excessively alarms me. Consider, brother, that ours is no upstart family: It is as ancient as the best in the kingdom: and. for several hundreds of years, it has never been known, that the heirs of it have disgraced themselves by unequal matches [...]. If you were descended from a family of yesterday, from one who is but a remove or two from the dirt you seem so fond of, that woukl be another thing. Let me tell you that I, and all mine, will renounce you for ever, if you can descend so meanly". RICHARIDON 1985, 293.

6 Cosi loriginale: "What a letter is this, my dear father and mother! One may see by it how poor people are clespised by the reach and the great! And yet we were all on a foot originally. Surely these proud people never think what a short stage life is; and that, with all their vanity, a time is coming, when they shall be on a level with us". Richarbson I985, $29+$.

- In inglese: "[...] to divert and entertain, and al the same time to instruct and improve the minds of the ron TH of both sexes; [...] to inculcate religion and morality; [...] set forth in the most exemplary lights, the parental, the filial, and the social duties, [...] to paint ITCE in its proper colours, to make it desemedly' odious; and to set VIRTLE in its own amiable light, to make it look lonely's. Richarison 1985, 31.

8 Che la sensilsilità per le distinzioni sociali costituisse un punto di discussione non irril- 
evante è mostrato anche da un altro aspento deella questione, che attiene in maniera direttal allambivalenza semantica del termine genere, e che mi piace entucleare qui attraterso il clialogo fra l'infuriata Lady Davers e Mr. 13., diakego che mostrat come anche la nobilitazione sociale sia sottomessa al categorie di genere, in quanto può atrenire per il solo tramite maschile. e non conosce reciprociti: "Supponi [...] che io avessi sposato il mozzo di stalla di mio padre! Che cosa avesti detto tu?" "Non atrei potuto compontami peggio [...] cli quanto roi arete fatto. Il rostro orgoglio, lady 1)arers, non ri consente di vedere nessuna differenzal nel caso che ponete?" "Néssunissima [...]. Che differenza può esserci fra un liglio di pezzente sposato con una signora, ce la figlia di un pezzente fatta sposa da un gentiluomo?" "Ve lo dirò io [...] la differenza è che l'uomo nobilital lit donna che prende. sia costei chiunque si roglia; e lakdotta nel proprio rango, qucilungue sia. Ma la donna, per quanto di nobili natali, si abbassa tramite un matrimonio vile, e clal proprio rango scende a quello di colui che si umilia a sposare. (Richarissox 1995, 52.4). Per la versione inglese dr. Ricianidsox $1985,440-41$.

9 Sempre nello stesso luogo delle Memorie, Goldoni aggiunge che ail publslico la trovò interessante e divertente; e di tutte le mie opere fino allora rappresentate fu quella che riportò la palma".

${ }^{10}$ Gia Norbert Jonard, parlando del dramma borghese e dei tipi di virtì messi in scena da Goldoni avera colto questa problematica del genere in maniera puntuale: “De fait, le choix d'un protagoniste vertueux conduit inévitablement au mélange des genres: le sérieux se mêle au présent, le touchant au comique". (Joxart) $19^{-7}, 545$ ).

11 Cito dalla Nota di Giuseppe Ortolani a La Pamela, in GoldoN 1939. III 1183.

12 Una risione dinsieme delle modifiche operate dia Goldoni sul testo del romanzo e un paragone clei singoli luoghi da questo divergenti nella sua ricluzione teatrale si legge nello studio di Bertolivo 82-116, al cap. IV': Goldoni and Richardson: Pamelas compared.

13 Colovizat -9 e 81 . La medesima ambiguita e teorizzata anche nel breve saggio di SCItLlo.

It Dal 1533 una legge stabiliva per i nobili che, se avessero sposato "alcuna fantescat o femina di villa, over qualunque altra di abjetta e vil condizionen avetbbero tolto ai figli il titolo nobiliare e i diritti politici e sociali ad esso connessi. Cli. MoLvinTI 314, che cita dall'Archivio di Stato. Del resto il governo reneziano era. su questo punto. di una sererità implacabile ancora negli anni in cui Goldoni scrivera la Pamela, come ben mostra l'esempio recato da Valsoakı) 114-15, n. 3: "Alla clata de] 5 maggio 1765 si può [...] leggere nelle Annotazioni degli Inquisitori di Stato (Archivio di Stato di Veneziá, anni 1-63-1-69) che una ballerina di nome Carlinat, che aveva spinto un giovane di famiglia patrizia fino allat "mostruosa decisione" di sposarlat, venne bandita in perpetuon. Vasussard cita inoltre

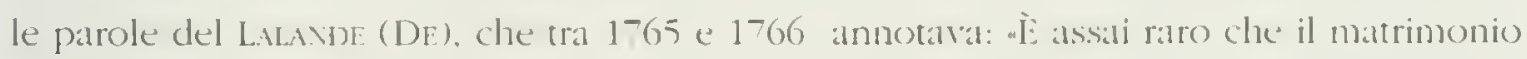
di un nobile veneziano con una cittadina sia approvato dal Maggior Consiglio e non se ne trova neanche un esempio tra le migliori famiglien. 
15 Nel secondo Settecento si modificáno profondamente sia la composizione del pul)blico di lettori - con un'ampia inclusione delle donne tra gli estimatori del genere romanzo - sia i modi e i tempi della lettura (entrambi fortemente vincolati a fattori quali le nuove condizioni di inurbamento e industrializzazione, la nuova gestione borghese del tempo e dei ruoli familiari, l'accresciuto potere d'acquisto e l'avvento di un'editoria di massa). La settecentesca "rivoluzione" della lettura è descritta con efficacia dalla teoria di Rolf Engelsing, secondo cui nel Settecento si assisterebbe al passaggio da una lettura di tipo intensivo (pochi testi, riletti con frequenza) ad una lettura caratterizzabile invece come estensiva (materiali sempre nuovi e diversi). Per una cliscussione critica di questa teoria si legga Wittmann. Un bell'esempio di ricostruzione della tipologia del lettore del diciottesimo secolo è dato, per il caso inglese, da J. P. Hunter, nel capitolo intitolato Readers Reading del suo fondamentale HUNTER 1990, 61-88.

${ }^{16}$ La prospettiva non cambia anche se volessimo tener conto del fatto che con ogni probabilità, dato l'uso corrente del tempo, Goldoni lesse Richardson per il tramite della diffusissima versione francese - falsamente (nu ripetutamente, anche oggi e ancora nella citata edizione nazionale della Pamela goldoniana, Goldoni 1995) attribuita all'Abbé Prévost, e non direttamente in originale: infatti gli studi più recenti di letteratura comparata hanno mostrato come - al di là della difficoltà di attribuzione della versione francese, il testo restituisse con una fedeltà quasi sconosciuta per i modelli mentali settecenteschi, l'originale inglese (cfr. in particolare il secondo capitolo del bel libro di Grafibr 46-70). Sarebbe estremamente interessante, ma richiedereble uno studio a sé, analizzare in maniera più approfonclita quali tratti distintivi del carattere della Pamela goldoniana si ritrovino già clelineati nella mediazione francese, e seguirne gli sviluppi.

17 E la già menzionata scenaı $1,13$.

18 Nell'originale: "As I have furnished you with a subject, I think I have a title to see how you manage it. Besides, there is such a pretty air of romance, as you tell your story, in yom plots, and my plots, that I shall be better directed how to wind up the catastrophe of the pretty novel". Richarison 1985, 268.

19 Goldoni rappresenta Pamela mentre scrive, o anche mentre consegna o legge lettere; tuttavia, sulla scena, questi appaiono piuttosto come stratagemmi per promuovere l'azione che non attimi di approfondimento psicologico, come erano invece nel romanzo.

${ }^{20}$ Cito qui il hel saggio di Bertoni 214 , e a questo studio faccio riferimento per tutto il paragrafo.

21 E nota, anche se ancora poco studiata, l'attività di romanziere di Chiari, e il cliretto e reciproco scambio intercorrente fra i romanzi (suoi e di altri) e la sua produzione teatrale; má sarà forse utile ricordare che lo stesso Goldoni mostra, in un epoca in cui sarebbe stato impossibile fare altrimenti, vari segni di interesse per il genere emergente del rorel: dalle Memorie (GolDon 1994, II 11 289) si evince ad esempio come egli scrivesse L Lincognita perseguitata contemporaneamente come commedia e come romanzo (incompiuto); a luj 


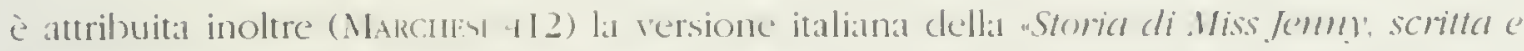
addirizzata dalla medesima a biledy contessa di Roscomond. Ambasciatrice della Corte di Francia in cunella di Danimarca. (Gomov 1-93).

22 II rileramento è in Cotomis. 90, n. 53.

23 Sulla lingua della Pamela goddoniana si veda lo studio di Fivetro.

24 Nelloriginale inglese: "You 1 on t. hussy! Do you know whom you speak to?" I lost all fear, and all respect. and said: "Yes, I do, sir. too well! Well may I forget that I am your servant, when you forget what belongs to a master." I solbled an cry most sadly. "What a foolish hussy you are!" said he: "Hase I done you any harm?" "Yes sir", said I, "the greatest harm in the world: You have taught me to forget myself, and what belongs to me: and have lessened the distance that fortune has made between us, by demeaning yourself, to be so free to a poor servant. Yet, sir, I will be hold to say. I am honest. though poor: And if you were a prince, I would not he otherwise than onest". Richiarimox 1985, 55.

25 Per la trafila erolutiva del personaggio. da Griselda alla Bettina della Putta onorata a Pamela, si reda lo studio di Bf.Niscrili.

26 Goldoni definisce la fanciulla per rapporto alla maritata. dicendola una "commedia da teatro" e non "da tavolino".

27 Cfr. Baretri 34-41 e 173-90: la Pamela è frustata nel n. XVII (1 giugno 1764, 464-71) e la Pamela marilata nel n. XXHI ( 15 agosto 1764, 601-20). La citazione è tratta da BARETTI 39.

28 Baretti non risparmia, a dire il rero, nemmeno la maritata: "Questa Pamela" spiega alla p. 173 aè una continuazione di cuell'altra Pamela sopranomatia fanciulla. che verso il fine della commedia fu già inaspettatamente trasformata di contadina in dama. perché. senza una tale trasformazione, il Goldoni non arrebbe avuto il coraggio di dare una fanciulla di vil sangue per moglie ad un cavaliere, quantunque l'accidente aresse riunite in tal fanciulla tanta bellezza e tante virtù. quante se ne possono immaginare. "Il decoro delle famiglie (dice il nostro galantuomo) non si cteve sagrificare al merito della virtù". Ecco una delle tante buone massime che s’imparano leggendo le opere di questi nostri illustri moderni! La virtù è una chimera. e la gente di gran prosapia deve badare assai più al sangue che non a quella. perché la virtù è cosa comunale, e se ne trovano delle carrettate in tutti i cantoni; che all'incontro il sangue nobile è una cosa rarissima, e da preferisi a turte quante le virtù del mondo. Vía. signori inglesi. venite a imparare la logica. la filosofia e la morale del nostro teatrale concittadino".

${ }^{29}$ L'ipotesi. che condivido pienamente. è avanzata in un contesto argomentativo diverso anche da BERTON 249: "La trasformazione del finale potrebbe però essere motivata non tanto da remore ideologiche e dal preoccupazioni contingenti quánto e soprattutto dalla logica intrinseca dellazione". 


\section{OPERE CITATE}

Baretti, Giuseppe. La finsta letteraria. A cura di Luigi Piccioni. Bari: Laterza, 1932. Beniscelli, Alberto. "Forza e delicatezza delle passioni. Le metamorfosi di Pamela." Studi Goldoniani. 8 (1988): 85-105.

Bertolino, Jane V. The Hamy Faces of Pamela. New York - Ottawa - Toronto: Legas, 1990.

Bertoni. Clotilde. "Dal romanzo alla scena: note intorno al personaggio femminile nella commedia settecentesca." Anmali dell Tstituto Italiano per gli Studi Storici. X (1987-1988): 213-74.

Cecioni, Cesare G. "Il romanzo inglese del Settecento: la tradizione picaresca e la nascita del romanzo sentimentale." Il romanzo: origine e sviluppo delle strutture narative nella letteratura occidentale. Pisa: ETS Editrice, 1987. 71-84.

Colombai, Orietta. "Dalla Pamela di Richardson alla Pamela goldoniana." Lingua e Letteratura. V (1987): 75-89.

Comparini, Lucie. "Pamela sur la scène italienne. Goldoni, Chiari, Cerlone." Les langues néo-latines. 279 (1991): 53-74.

Finetto, Annamaria. "La Pamela e La buona figlitola: il linguaggio patetico di Goldoni." Studi Goldoniani. 8 (1988): 107-36.

Folkenflik, Rohert. "Pamela: Domestic Servitude, Mariage and the Novel." Eighteenth Century' Fiction. V 3 (1993): 25.3-68.

Goldoni, Carło. Storia di Wiss Jenny, scritta e addirizzata dalla medesima a Miledy contessa di Rosconond. Ambasciatrice della Corte di Francia in quella di Danimarca. Opera di Madama Riccohoni, celebre autrice francese. Traduzione arhitraria del Sig. arvocato Carlo Goldoni. 2 tomi. Venezia: Antonio Curti di Giacomo, 1793.

Goldoni. Carlo. Memorie. Con minappendice di scritti goldoniani. Trad. di Guido Davico Bonino. Torino: Einaudi, 1994. ( 1á ed. 1967).

Goldoni. Carlo. Pamela fancinlla - Pamela maritata. A cura di Ilaria Crotti. Venezia: Marsilio, 1995. 379-453.

Goldoni, Carto. Tutte le opere. A cura di Giuseppe Ortolani. Milano: Mondadori, 1939. Grateber. Wilhelm. Der englische Roman in Frankreicb: 1741-1763. L̈bersetzungsgeschichte als Beitrag zur fianzösischen Literaturgeschichte. Heidelberg: Universitätsverlag C. Winter, 1995.

Grantham Turner, James. "Novel Panic: Picture and Performance in the Reception of Richardson Pamela." Representations 48 (1994): 70-96.

Iunter, I. Paul, Before Notels: The Cultural Contexts of Eighteentb Century, Englisb Fiction. New York - London: W. W. Norton \& Company, 1990.

Jonard. Norbest. "Le foumal étranger comme intermediaire en France de la littéra- 
ture italienne." Rerne de littérature cemparée. (1965) oct.-dée.: 586-8s.

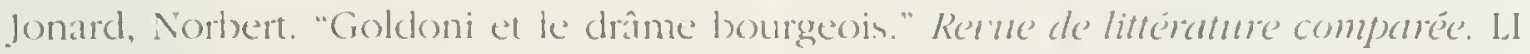
$4\left(19^{-7}\right): 536-52$.

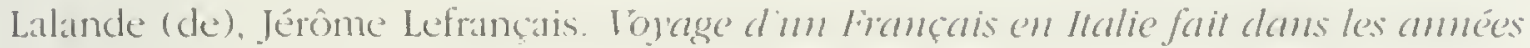
1765 (17 1766.8 voll. Paris: 1768.

Marchesi, Giambattista. Romanzierie romanzi del Settecento. Manziana: Vecchiarelli, 1991. (anastatica delled. 1903).

Molmenti. Pompeo. Storia di lenezia nella tita prinala. Trieste: Lint, 1973. ( ${ }^{\text {a }}$ ed. $1882)$.

Richardson, Samuel. Pamela, or Tintme Rendarded. Edited by Peter Sabor, with an introduction by Margatreth Anne Doody: London: Penguin 1985.

Richardson. Samuel. Pamela o la l'irti ricompensata. Trad. italiana di Masolino DAmico. [Milano]: Frassinelli, 1995.

Sciullo, Luciana. "Pamela da Richardson a Goldoni". Quaderni di lingue eletteratme. (1976): $11^{7}-21$.

Segre, Cesare. Anvamento allanalisi del testo lettermio. Torino: Einaudi. 1985. 23463.

Toldo, Pietro. "Attinenze fra il teatro comico di Voltatire e quello di Goldoni." Giornale Storico della Letteratura italiana. XXXi ( 1898): 343-60.

Vaussard. Maurice. La t'ita quotidiana in Italia nel Settecento. Milano: Rizzoli. 1990. ( $1^{\mathrm{a}}$ ed. 1959).

Wittmann, Reinhard. "Lna 'rivoluzione della lettura" alla fine del XT 711 secolo?". Storia della lettura nel mondo occidentale. A cura di Guglielmo Cavallo e Roger Chartier. Bari: Laterza, 1995. 337-69. 


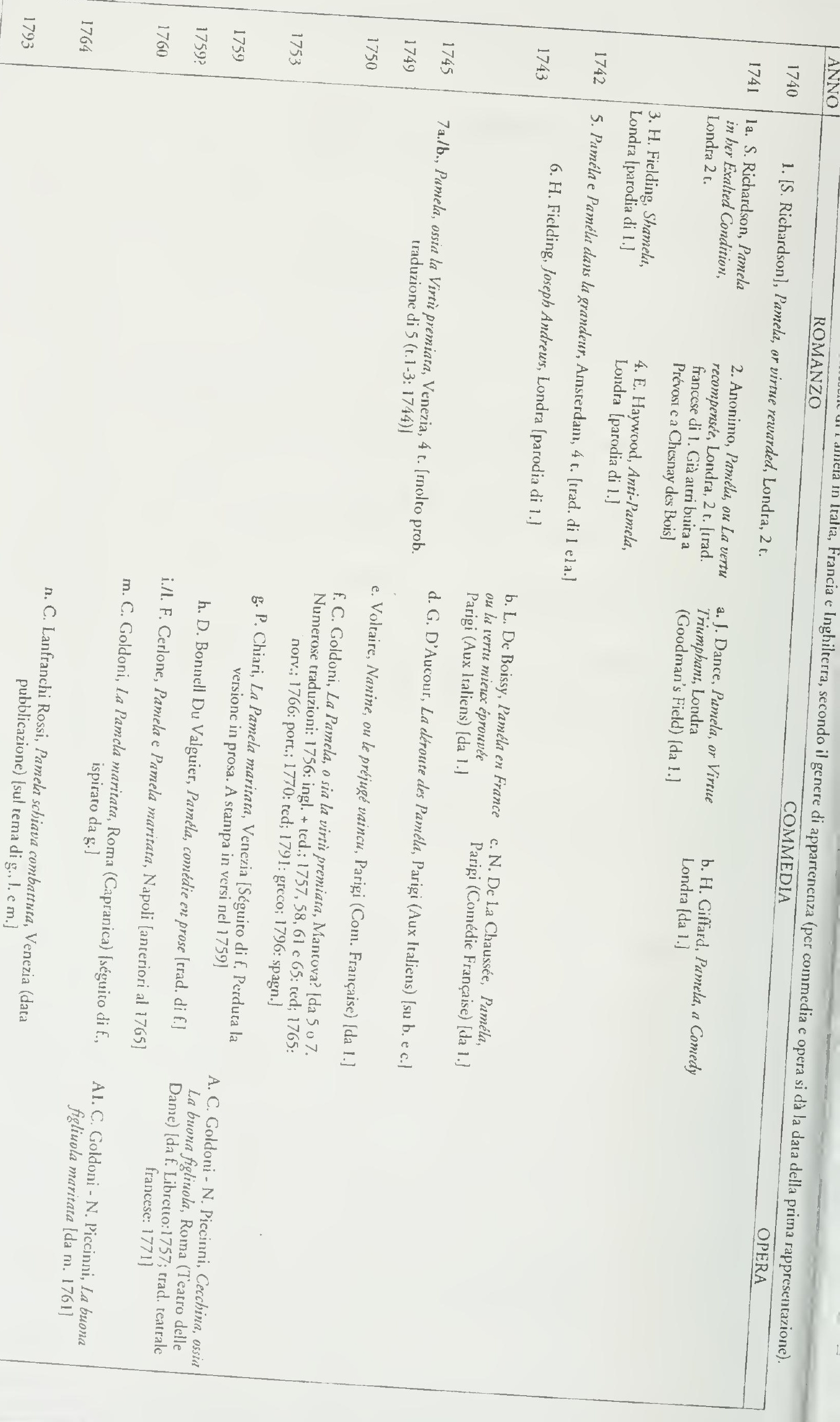

